\title{
Trastornos hereditarios de la coagulación en adolescentes con sangrado menstrual excesivo, ¿debemos evaluar la vía fibrinolítica?
}

\author{
Inherited bleeding disorders in adolescents with excessive menstrual bleeding. \\ Should we evaluate the fibrinolytic pathway?
}

\author{
N. Aguirre ${ }^{a}$, J. Pereira ${ }^{b}$, F. Barriga ${ }^{a}$, MA. Wietstruck ${ }^{a}$, O. Panes ${ }^{c}$, P. Sepúlvedad ${ }^{d}$, A. Salgado ${ }^{a}$, P. Zúñiga ${ }^{a}$
}

aMédico, Hemato-Oncólogo Pediatra. Departamento de Pediatría. Pontificia Universidad Católica de Chile. Santiago, Chile

bMédico Hematólogo. Departamento de Hematología. Pontificia Universidad Católica de Chile. Santiago, Chile

'Bioquímico. Laboratorio de Hemostasia. Pontificia Universidad Católica de Chile. Santiago, Chile

¿Médico Hemato-Oncólogo Pediatra. Unidad de Oncología Hospital del Talca. Talca, Chile

Recibido: 26 de diciembre de 2019; Aceptado: 5 de abril de 2020

¿Qué se sabe del tema que trata este estudio?

El sangrado menstrual excesivo (SME) afecta al $40 \%$ de las adolescentes. Los trastornos hereditarios de la coagulación (THC) son la causa secundaria más frecuente en este grupo etario. En muchos casos puede ser el primer síntoma, determinando que sea una oportunidad diagnóstica.
¿Qué aporta este estudio a lo ya conocido?

Este estudio nos da una aproximación en cuanto a la prevalencia de los trastornos hereditarios de la coagulación en adolescentes chilenas con SME y explora la posible participación de una alteración de la fibrinólisis.

\section{Resumen}

El Sangrado Menstrual Excesivo (SME) es un problema frecuente en la adolescencia. La prevalencia de trastornos hereditarios de la coagulación (THC) como causa del SME no está bien establecida y la participación de defectos de la vía fibrinolítica ha sido poco explorada. Objetivo: Determinar la prevalencia de THC y defectos de la fibrinólisis en adolescentes con SME. Pacientes y Método: Se incluyeron 93 adolescentes, edad 11 a 18 años. Los antecedentes personales y familiares de sangrado se obtuvieron con un cuestionario estandarizado. Se controló exámenes: tiempo de protrombina (TP), tiempo de tromboplastina parcial activada (TTPa), estudio del factor Von Willebrand, recuento y función plaquetaria. Los pacientes que no fueron diagnosticados como THC, se evaluaron adicionalmente con el tiempo de lisis del coágulo. Resultados: 41 pacientes (44\%) fueron diagnosticados como THC: Enfermedad de Von Willebrand $\mathrm{n}=28$, defectos de la función plaquetaria $\mathrm{n}=$ 8 , hemofilia leve $\mathrm{n}=5$. Se confirmó disminución del tiempo de lisis del coágulo en 31 pacientes. El $54 \%$ de pacientes diagnosticado como THC, tuvo SME como la primera manifestación hemorrágica. Conclusión: Estos resultados apoyan la necesidad de evaluación de la coagulación, incluyendo la vía fibrinolítica, en el estudio de adolescentes con SME.
Palabras clave: Sangrado menstrual excesivo; adolescentes; fibrinólisis; BUC; sangrado uterino anormal; trastornos hereditarios de la coagulación; sangrado menstrual abundante

Correspondencia:

P. Zúñiga

pzuniga@med.puc.cl 


\section{Abstract}

Heavy Menstrual Bleeding (EMB) is a frequent problem in adolescence. The prevalence of inherited bleeding disorders (IBD) as a cause of EMB is not well established and the involvement of fibrinolytic pathway defects has been poorly explored. Objective: To determine the prevalence of IBD and fibrinolysis defects in adolescents with EMBs. Patients and Method: 93 adolescents (11 to 18 years old) were included. Personal and family history of bleeding were obtained through a standardized questionnaire. The following lab tests were performed: prothrombin time (PT), activated partial thromboplastin time (aPTT), von Willebrand factor quantification, and platelet count and function. Those patients who were not diagnosed with IBD were further evaluated with clot lysis time assay. Results: 41 patients (44\%) were diagnosed as IBD (Von Willebrand disease $n=28$, platelet function defects $n=8$, mild hemophilia $n=5$. Decreased clot lysis time was found in 31 patients. $54 \%$ of patients diagnosed with IBD had EMB as the first hemorrhagic manifestation. Conclusion: These results support the need to evaluate the coagulation process, including the fibrinolytic pathway in the study of adolescents with EMB.

\section{Keywords:}

excessive menstrual bleeding; adolescents; fibrinolysis; BUC; abnormal uterine bleeding; inherited bleeding disorders; heavy menstrual bleeding

\section{Introducción}

El SME se define como un sangrado prolongado $>7$ días y/o flujos superiores a $80 \mathrm{ml}$ de pérdida de sangre $^{1-6}$. Recientemente el National Institute for Health and Care Excellence (NICE) en el Reino Unido, lo ha definido como excesiva pérdida de sangre menstrual que interfiere con la calidad de vida, física, emocional, social y material de la mujer, abordando de manera más integral el problema ${ }^{7,8}$.

Afecta aproximadamente al $40 \%$ de las adolescentes ${ }^{1,9}$ causando morbilidad y en algunos casos, mortalidad, además de afectar las actividades de la vida diaria, alterando la calidad de vida ${ }^{4,10-12}$. El buen diagnóstico del SME permite establecer medidas activas de manera oportuna para reducir el riesgo de transfusiones, anemia crónica, ausentismo escolar y mejorar calidad de vida ${ }^{13-16}$.

Para mejorar el diagnóstico del SME, desde el 2006, la Academia Americana de Pediatría y el Colegio Estadounidense de Ginecología y obstetricia han recomendado considerar las características de la menstruación como un "signo vital" en la evaluación médica de niñas y adolescentes ${ }^{17-19}$.

La causa más frecuente del sangrado uterino anormal en el periodo postmenárquico es la inmadurez del eje hipotalámo-hipófisis-ovario, que conduce a ciclos anovulatorios $^{2,20-22}$. La prevalencia de trastornos hereditarios de la coagulación muestra variaciones en los estudios publicados, especialmente en EvW con un intervalo entre un $5 \%$ a $36 \%$ y la DP entre un $3 \%$ a un $68 \%$ de las pacientes con $\mathrm{SME}^{23-25}$.

Los trastornos de coagulación hereditarios graves si bien, tienen como manifestación SME, estarán diagnosticados previo a la menarquia y tienen la mayor parte de las veces, un manejo multidisciplinario, desde etapas tempranas de la vida.
El mayor desafío diagnóstico son las coagulopatías leves o Mild Bleeding Disorders (MBD), ya que pueden presentar síntomas leves o inaparentes, hasta que se presenta un desafío hemostático, como un trauma o cirugía o en este caso, la menstruación.

Dentro de las llamadas coagulopatías leves o MBD se encuentran la Enfermedad de von Willebrand, Disfunciones plaquetarias leves y los déficit leves de factores de coagulación.

El diagnóstico de laboratorio de los MBD ha sido discutido ${ }^{26} \mathrm{y}$ recientemente se han estandarizado criterios diagnósticos para las enfermedades de hemostasia primaria, incluida la enfermedad de Von Willebrand y los trastornos de la función plaquetaria. Nuestro laboratorio ha formado parte de este trabajo y se han adoptado sus recomendaciones ${ }^{14,26}$.

Los síntomas de los MBD corresponden mayormente a sangrado mucocutáneo o desproporcionado al estímulo, en caso de ser expuesto a un desafío hemostático como trauma o cirugía. Como una forma de objetivar estos síntomas se han creado cuestionarios como el ISTH-SSC Bleeding Assessment Tool (BAT), que ha

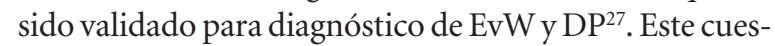
tionario consta de varios puntos para evaluar distintos síntomas de sangrado incluyendo el sangrado menstrual excesivo. Este cuestionario es positivo en niños, cuando se obtiene un puntaje mayor o igual a 3 puntos, lo cual se cumple, por ejemplo, en aquellas pacientes con SME que han requerido uso de antifibrinolíticos, tratamiento hormonal, hospitalización o trasfusiones ${ }^{27}$.

En este sentido, se ha descrito que el $92 \%$ de las pacientes con THC presentan $\mathrm{SME}^{28}$, por lo que consideramos que la menarquia puede ser una oportunidad diagnóstica para estas pacientes, permitiendo un mejor manejo, no solo para su SME, si no que para futuras intervenciones tales como cirugías mayores y menores y durante el embarazo y parto. 
Tabla 1. Resultados

\begin{tabular}{|c|c|c|c|c|c|c|c|}
\hline & $\mathrm{EvW}^{\mathrm{a}}$ & $\begin{array}{l}\text { Defectos de } \\
\text { la fibrinólisis }\end{array}$ & $\begin{array}{l}\text { Disfunción } \\
\text { plaquetaria }\end{array}$ & $\left.\mathrm{PT}\right|^{\mathrm{b}}$ & $\begin{array}{l}\text { Déficit de } \\
\text { factores }\end{array}$ & $B \cup C^{c}$ & Total \\
\hline $\mathrm{N}$ & 28 & 31 & 8 & 1 & 5 & 20 & 93 \\
\hline ISTH-BAT positivo & 20 & 26 & 6 & 1 & 4 & 15 & 72 \\
\hline Historia familiar de sangrado & 18 & 19 & 4 & 0 & 4 & 10 & 55 \\
\hline Sangrado menstrual excesivo como primer síntoma & 15 & 9 & 3 & 0 & 4 & 8 & 39 \\
\hline Anemia & 9 & 16 & 3 & 0 & 0 & 7 & 35 \\
\hline
\end{tabular}

No está totalmente claro el papel de la fibrinólisis en la patogenia del SME, a pesar que la mayoría de las guías recomiendan antifibrinolíticos dentro de su manejo. Nuestro laboratorio ha desarrollado una técnica global de evaluación de la fibrinólisis ${ }^{29}$ llamado tiempo de lisis del coagulo, la cual hemos incorporado en la evaluación de los pacientes con sangrado mucocutáneo, especialmente sangradores de causa desconocida [Bleeding unknow cause (BUC)]. El objetivo de este estudio fue determinar la prevalencia de trastornos hereditarios de la coagulación y aceleración del tiempo de lisis de coagulo, en pacientes derivados por SME a policlínico hematología.

\section{Pacientes y Método}

Se diseñó un estudio descriptivo y transversal que incluyó pacientes de 11 a 18 años de edad que consultaron por SME en el policlínico de Hematología Pediátrica de la Pontificia Universidad Católica de Chile desde abril de 2016 hasta septiembre de 2017. Se excluyó a los pacientes que recibían terapia anticoagulante y que tuvieran diagnóstico de un trastorno hereditario de la coagulación previamente.

Todos los pacientes fueron encuestados por síntomas de sangrado personal utilizando el cuestionario de ISTH-SSC Bleeding Assessment Tool y por antecedentes familiares de síntomas de sangrado. Una puntuación de $>0=3$ se consideró positiva.

Las pruebas generales de coagulación que incluyen el tiempo parcial de tromboplastina (TTPa), el tiempo de protrombina (TP) y el hemograma completo se realizaron en la primera visita. Se solicitó el estudio de EvW que incluye FVIII coagulante, FvW Antigénico, Co factor ristocetina, prueba de unión a colágeno y evaluación de la función plaquetaria con la prueba de agregación plaquetaria. Se hizo énfasis a las pacientes que para realizar los exámenes debían estar sin cuadros infecciosos intercurrentes y no haber consumido anti- inflamatorios no esteroidales al menos 15 días previos a la toma de muestra.

Aquellas pacientes con TP-TTPa alterado y aquellas que tuvieron antecedentes familiares de trastornos hereditarios, fueron estudiadas con factores de la coagulación.

Aquellas pacientes con agregación plaquetaria alterada, además fueron estudiadas con secreción plaquetaria.

Si todos estos resultados fueron normales, el estudio se complementó con la prueba del tiempo de lisis del coágulo para evaluar la fibrinólisis. Nuestro laboratorio utiliza plasma rico en plaquetas estimulado con ristocetina o agonista del receptor de trombina 6, y plasma pobre en plaquetas, evaluando los defectos del sistema fibrinolítico en una sola técnica ${ }^{28}$.

Aquellos pacientes con puntaje positivo por ISTHBAT y aquellos con antecedentes familiares de sangrado, se correlacionaron con los diagnósticos de trastornos de la coagulación (EvW, defectos de factores de coagulación, DP) y defectos de la fibrinólisis. A través de análisis estadístico por IBM SPSS Statistics 20.0.1.

El estudio fue aprobado por el comité de ética de la Pontificia Universidad Católica de Chile, utilizando consentimiento informado.

\section{Resultados}

Se incluyeron 93 pacientes. La mediana de edad fue de 14 años, con un rango entre 11 y 18 años.

Setenta y dos pacientes obtuvieron una puntuación en el cuestionario de síntomas de sangrado BAT positiva. De estos pacientes el 54\% no presentaba síntomas de sangrado previo a su menarquía.

Se encontró antecedentes familiares de síntomas de sangrado en 55 pacientes.

De los exámenes iniciales, 80 pacientes $(90 \%)$ tenían TP y TTPa normales y 35 (38\%) pacientes presentaron anemia, siendo grave (hemoglobina $<7 \mathrm{~g} / \mathrm{dl}$ ) en 6 casos. 
Veintiocho pacientes fueron diagnosticados con $\mathrm{EvW}, 8$ con defectos de la función plaquetaria, 5 con deficiencia de factor de coagulación (3 pacientes con deficiencia de factor VIII, uno con deficiencia de factor IX y uno con deficiencia de factor XI) y 1 paciente con trombocitopenia inmune primaria (tabla 1).

El análisis de fibrinólisis se realizó en los 51 pacientes con un estudio de coagulación normal y 31 mostraron un tiempo de lisis del coágulo acelerado, lo que es compatible con un estado de hiperfibrinolisis. Combinando pacientes con trastornos hereditarios de la coagulación y defectos de la fibrinólisis, 72 pacientes $(77 \%)$ tenían algún tipo de trastorno hemorrágico. En 15 pacientes $(75 \%)$ diagnosticados con EVW, el SME fue el primer síntoma de la enfermedad.

No se encontró correlación entre BAT positivo o los antecedentes familiares y la presencia de un trastorno hereditario de la coagulación, incluidos los defectos de la fibrinólisis.

\section{Discusión}

Si bien este es un grupo seleccionado, ya que ha sido derivado por sospecha de coagulopatía o por anemia, la prevalencia de THC resultó ser alta. Esto apoya lo descrito en la literatura y refuerza la idea de que la posibilidad de un trastorno hereditario de la coagulación, debe estar presente cuando se evalúa a una adolescente con SME.

Aunque no fue objetivo de este trabajo evaluar las causas de derivación, pensamos que es importante, que el pediatra y el personal de salud que atiende adolescentes, incluyan dentro de la evaluación, las características de la menstruación y que tomen un rol activo en el diagnóstico, tratamiento, seguimiento y en caso necesario, derivación a especialista, de las adolescentes con SME.

Es importante destacar que casi el $90 \%$ de las pacientes estudiadas tenían las pruebas de estudio que se solicitan con más frecuencia para descartar los trastornos hemorrágicos (TP-TTPa), normales. Lo cual refuerza que estos exámenes normales no excluyen la presencia de coagulopatía y en el caso que existan otros sangrados mucocutáneos, antecedentes familiares o mala respuesta a tratamiento debiera derivarse para estudio de THC.

La prevalencia de la EVW en la población general es baja $(<1 \%)$. Se ha informado una incidencia de 5 a $36 \%$ en adolescentes con $\mathrm{SME}^{26}$. En nuestro estudio, la EVW se diagnosticó en el 30\% de los pacientes y en la mayoría de ellas, el SME fue el primer síntoma de la enfermedad, por lo tanto, el estudio de SME podría ser una oportunidad diagnóstica para estas adolescentes.
La incidencia informada de defectos de la función plaquetaria en pacientes con SME también muestra una amplia variación, entre el $3 \%$ y el $68 \%^{9,24,30}$. En nuestra serie el $9 \%$ de los pacientes tenían defectos de la función plaquetaria, sin embargo, no todas contaban con estudio de secreción plaquetaria, que podría haber agregado pacientes que tengan sólo defectos de secreción ${ }^{28}$.

Según estudio de Quiroga y cols. en población chilena $^{28}$, un $59,6 \%$ de los pacientes corresponden a sangradores de causa desconocida (BUC), lo cual es coincidente con el 54\% de las pacientes en que no encontramos THC. Creemos que estas pacientes debieran permanecer en seguimiento, tanto para evaluar repercusiones secundarias de SME como para ver su comportamiento ante desafíos hemostáticos futuros ${ }^{28}$.

De los pacientes de nuestro estudio catalogados inicialmente como BUC, el 60\% mostró un acortamiento del tiempo de lisis del coágulo lo cual corresponde al $33 \%$ del grupo total. Esto hace pensar que los defectos globales de la fibrinólisis pueden jugar un papel en SME.

Existe evidencia de que la fibrinólisis participa en el ciclo endometrial. Los niveles de activadores de plasminógeno, especialmente el activador de plasminógeno de tipo tisular (t-PA) y su inhibidor, el inhibidor-1 del activador de plasminógeno (PAI-1), aumentan significativamente en la fase secretora tardía del endometrio. En pacientes con sangrado uterino anormal, se ha descrito una mayor actividad fibrinolítica en el flujo menstrual, además, se ha informado que las mujeres que reciben terapia hormonal muestran una disminución de la actividad fibrinolítica endometrial ${ }^{31,32}$. S. Wiewel-Verschueren y cols publicaron un estudio en pacientes adultas con SME midiendo cuantitativamente los activadores e inhibidores de la fibrinólisis y no encontraron alteraciones evidentes $^{33}$. Sin embargo, pensamos que una prueba global como la que usamos en nuestras pacientes puede describir mejor las interacciones del sistema fibrinolítico y su resultado final.

\section{Conclusión}

La prevalencia de THC fue alta en el grupo estudiado. El SME fue el primer síntoma en un grupo importante de pacientes con EvW, lo cual refuerza la idea de que el SME es una buena oportunidad diagnóstica para THC, aún en pacientes con escasa o nula presencia de otros síntomas de sangrado.

Nos parece que la alta incidencia de acortamiento del tiempo de lisis del coágulo encontrados en este estudio, orienta a que es necesaria la evaluación de la vía fibrinolítica en las adolescentes con SME. 


\section{Responsabilidades Éticas}

Protección de personas y animales: Los autores declaran que los procedimientos seguidos se conformaron a las normas éticas del comité de experimentación humana responsable y de acuerdo con la Asociación Médica Mundial y la Declaración de Helsinki.

Confidencialidad de los datos: Los autores declaran que han seguido los protocolos de su centro de trabajo sobre la publicación de datos de pacientes.
Derecho a la privacidad y consentimiento informado: Los autores han obtenido el consentimiento informado de los pacientes y/o sujetos referidos en el artículo. Este documento obra en poder del autor de correspondencia.

\section{Conflicto de intereses}

Los autores declaran no tener conflicto de intereses.

\section{Referencias}

1. Haamid F, Sass A, Dietrich J. Heavy Menstrual Bleeding in Adolescents. J Pediatr Adolesc Gynecol. 2017;30(3):33540.

2. Seravalli V, Linari S, Peruzzi E, et al. Prevalence of Hemostatic Disorders in Adolescents with Abnormal Uterine Bleeding. J Pediatr Adolesc Gynecol. 2013;26(5):285-9.

3. Nowak-Göttl U, Kenet G. Challenging Aspects of Managing Hemostasis in Adolescents. Acta Haematol (2014);132:326-30.

4. Bricaire L, Laroche E, Christin-Maitre S. Meno-metrorrhagia, dysmenorrhea in adolescents. Archives de pédiatrie. 2013;20:910-4.

5. Graham RA, Davis JA, Corrales-Medina FF. The Adolescent with Menorrhagia: Diagnostic Approach to a Suspected Bleeding Disorder. Pediatr Rev. 2018;39(12):588-600.

6. Powers JM, Stanek JR, Srivaths L, Haamid FW, O’Brien SH. Hematologic Considerations and Management of Adolescent Girls with Heavy Menstrual Bleeding and Anemia in US Children's Hospitals. J Pediatr Adolesc Gynecol. 2018;31(5):446-450.

7. Magnay J, O'Brien S, Gerlinger C Seitz C. A systematic review of methods to measure menstrual blood loss. BMC Women's Health. 2018;18, Article number: 142.

8. Borzutzky C, Jaffray J. Diagnosis and Management of Heavy Menstrual Bleeding and Bleeding Disorders in Adolescents. JAMA Pediatr. 2020;174(2):186-94.

9. Amesse L, Pfaff-Amesse T, Gunning W, et al. Clinical and laboratory characteristics of adolescents with platelet function disorders and heavy menstrual bleeding. Exp Hematol Oncol 2013;2(1):3.

10. Torres R, Zajer C, Menéndez M, et al. El sangrado menstrual excesivo afecta la calidad de vida en adolescentes. Rev Chil Pediatr. 2017;88(6):717-22.

11. Alaqzam TS, Stanley AC, Simpson PM,
Flood VH, Menon S. Treatment Modalities in Adolescents who present with heavy menstrual bleeding. J Pediatr Adolesc Gynecol. 2018;5:451-8.

12. Karaman K, Ceylan N, Karaman E, et al. Evaluation of the Hemostatic Disorders in Adolescent Girls with Menorrhagia: Experiences from a Tertiary Referral Hospital. Indian J Hematol Blood Transfus. 2016;32(3):356-61.

13. Kouides PA, Kadir RA. Menorrhagia associated with laboratory abnormalities of hemostasis: epidemiological, diagnostic and therapeutic aspects. Journal of Thrombosis and Haemostasis. 2007;5 (Suppl. 1):175-82.

14. MacEachern K, Kaur H, Toukh M, et al. Comprehensive Evaluation of Hemostasis in Normal Women: Impact on the Diagnosis of Mild Bleeding Disorders. Clinical and Applied Thrombosis/ Hemostasis 2015;21(1):72-81.

15. Bevan JA, Maloney KW, Hillery CA, Gill JC, Montgomery RR, Scott JP Bleeding disorders: A common cause of menorrhagia in adolescents. J Pediatr. 2001;138(6):856-61.

16. Lukes AS, Kadir RA, Peyvandi F, Kouides PA. Disorders of hemostasis and excessive menstrual bleeding: prevalence and clinical impact. Fertility and Sterility. 2005;84(5):1338-44.

17. Committee on Adolescent Health Care. Menstruation in girls and adolescentes: Using the menstrual cycle as a vital sign. Committe Opinion No 651, Dec 2015.

18. Schulin-Zeuthen C, Conejero C. Trastornos menstruales y dismenorrea en la adolescencia. Rev Med Clin Condes 2011;22(1):39-47.

19. Díaz A, Laufer M, Breech L. Menstruation in girls and adolescents: Using the menstrual cycling as a vital sign. Pediatrics. 2006;118:2245-50.

20. Dowlut-McElroy T, Williams KB, Carpenter SL, Strickland JL. Menstrual Patterns and Treatment of Heavy Menstrual Bleeding in Adolescents with Bleeding Disorders. J Pediatr Adolesc
Gynecol. 2015;28(6):499-501.

21. Mullins TL, Miller RJ, Mullins ES. Evaluation and Management of Adolescents with Abnormal Uterine Bleeding. Pediatric Annals. 2015;44(9):e218-22.

22. Kouides PA, Conard J, Peyvandi F, Lukes A, Kadir R. Hemostasis and menstruation: appropriate investigation for underlying disorders of hemostasis in women with excessive menstrual bleeding. Fertility and Sterility. 2005;84(5):1345-51.

23. Edlund M, Blombäck M, von Schoultz B, Andersson O. On the value of menorrhagia as a predictor for coagulation disorders. Am J Hematol. 1996;53(4):234-8.

24. Mumford AD, Frelinger AL, 3rd, Gachet $\mathrm{C}$, et al. A review of platelet secretion assays for the diagnosis of inherited platelet secretion disorders. Thromb Haemost. 2015;114(01):14-25.

25. Ayesha Z, Madhvi R. Challenges of diagnosing and managing the adolescent with heavy menstrual bleeding. Thrombosis Research. 2016;143:91-100.

26. Rodeghiero F, Mezzano D, Quiroga $\mathrm{T}$, Zúñiga P. Diagnosing type 1 von Willebrand disease: good for patient's health or for doctor's prestige?: comment. Journal of Thrombosis and Haemostasis. 2014;12:2131-9.

27. https://bleedingscore.certe.nl/

28. Quiroga T, Goycoolea M, Panes O, et al. High prevalence of bleeders of unknown cause among patients with inherited mucocutaneous bleeding. A prospective study of 280 patients and 299 controls. Haematologica. 2007;92(3):357-65.

29. Panes O, Padilla O, Matus V, et al. Clot lysis time in platelet-rich plasma: Method assessment, comparison with assays in platelet-free and platelet-poor plasmas, and response to tranexamic acid. Platelets. 2012;23(1):36-44.

30. Mills HL, Abdel-Baki MS, Teruya J, et al. Platelet function defects in adolescents with heavy menstrual bleeding. Haemophilia. 2014;20(2):249-54.

31. Gebhart J, Kepa S, Hofer S, et al. 
Fibrinolysis in patients with a mild-to-moderate bleeding tendency of unknown cause. Ann Hematol. 2017;96(3):489-95.

32. Koh SC, Singh K. The effect of levonorgestrel-releasing intrauterine system use on menstrual blood loss and the hemostatic, fibrinolytic/inhibitor systems in women with menorrhagia. J Thromb Haemost. 2007;5(1):133-8.
33. Wiewel-Verschueren S, Knol HM, Lisman $\mathrm{T}$, et al. No increased systemic fibrinolysis in women with heavy menstrual bleeding. Journal of Thrombosis and Haemostasis. 2014;12:1488-93. 